\title{
ENGAGEMENT-POLITIK
}

\section{Mehr Respekt bitte}

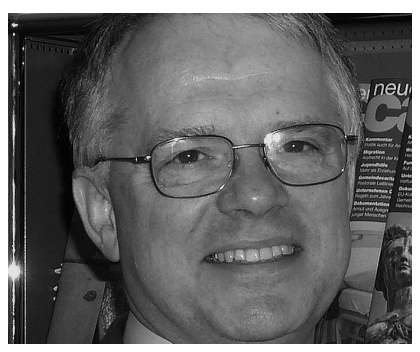

VON MARIO JUNGLAS

Der Theologe und Jurist ist Leiter des Berliner Büros des Deutschen Caritasverbandes e. V.

E-Mail Mario.Junglas@caritas.de
Engagementpolitik hat zurecht Konjunktur. Doch wer Demokratie nicht nur als eine Regierungsform, sondern zunächst als eine Lebensform sieht, der muss sich auch um Menschen und Gruppen kümmern, die sich dem gängigen bürgerschaftlichen Engagement scheinbar verweigern.

Das Konzept des bürgerlichen Engagements und der Zivilgesellschaft mag in Deutschland vielleicht noch nicht die Leitidee der Politik und des Regierungshandelns sein (1), es ist nach einem langen Anlauf aber dabei, sich zu einem eigenständigen Politikfeld zu entwickeln.

Diese Entwicklung reicht von den Freiwilligensurveys 1999 und 2004 über die Enquetekommission des Bundestages 2002 "Zukunft des bürgerschaftlichen Engagements ", die Einrichtung des Unterausschusses Bürgerschaftliches Engagement 2003, die Initiative "ZivilEngagement Miteinander - Füreinander « und weitere Stufen bis zum Koalitionsvertrag zwischen CDU, CSU und FDP vom Oktober 2009, in dem von einer nationalen Engagementstrategie, einem Gesetz zur Förderung des bürgerschaftlichen Engagements und bundeseinheitlichen Förderinstrumenten die Rede ist.

Solche Engagementpolitik versteht das Bundesministerium für Familie, Senioren, Frauen und Jugend als ein innovatives Politikfeld, eine gesellschaftliche Querschnittsaufgabe und hält dessen Etablierung für das notwendige Ergebnis gesellschaftlicher Entwicklungen und Herausforderungen sowie politischer Entscheidungen. (2)

Die gesellschaftlichen Herausforderungen sind nach der Überzeugung vieler Autoren der demografische Wandel und die damit verbundenen Veränderungen in der Altersstruktur, die schleichenden Veränderungen der Grundlagen des Zusammenlebens in unserer Gesellschaft (3), der erhöhte Pflegebedarf, zunehmende Individualisierung, soziale Desintegration und die schwindende Leistungsfähigkeit traditioneller Sozialsysteme. (4)

Zwar wird immer wieder betont, dass es weder Aufgabe noch Funktion des bürgerschaftlichen Engagements und der Zivilgesellschaft sei, den Lückenbüßer für einen
Sozialstaat zu spielen, der an seine Ressourcen-Grenzen stößt oder aus welchen Gründen auch immer seine Leistungen einschränkt. Ebenso deutlich ist, dass die sich auftuenden Lücken das Nachdenken über zivilgesellschaftliches Engagement mindestens befördern. Wenn die Bereiche Gesundheit, Soziales, Bildung und Kultur als Felder bürgerschaftlichen Engagements beschrieben werden und ihm wichtige Funktionen bei der Realisierung staatlicher sozialpolitischer Aufgaben zugemessen werden (5), dann steckt hierin einerseits eine Anerkennung dieses Engagements, andererseits wird aber auch die klare Erwartung an Leistungen und Lösungen deutlich.

Dem entspricht, dass Engagementpolitik vor allem als eine Politik der Potenziale verstanden wird: Die Potenziale nahezu aller Bürgerinnen und Bürger sollen gehoben werden, die Potenziale der älteren Generation ebenso wie die der Jugend und der Migrantinnen und Migranten. Es wird mit Potenzialen für die Demokratie und für die soziale Integration ebenso gerechnet wie für die Wohlfahrtsproduktion. Durch effektivere Strukturen, Prozesse und Rahmenbedingungen sollen Hervorbringung und Nutzung dieser Potenziale erleichtert werden. Die genannten Potenziale werden als Werte beschrieben, die es wie einen Schatz zu heben gilt und die einen entscheidenden Beitrag zur gesellschaftlichen Entwicklung, aber auch zum Erhalt des Erreichten leisten können. Demokratie stabilisierende und Werte erhaltende Funktionen werden dem bürgerschaftlichen Engagement ebenso zugeschrieben wie innovative Wirkungen. (6) Die Zivilgesellschaft wird so zur Stütze der gesellschaftlichen Integration, aber auch des wirtschaftlichen Wachstums, des Wohlstands und stabiler demokratischer Strukturen. (7)

Neben der sozialen Integration und der Kompensation von Leistungen wird die Partizipation als ein Ziel bürgerschaftlichen Engagements beschrieben, auch wenn die Potenzial-Orientierung offenbar die Versuchung nährt, Partizipation eher als die Möglichkeit zum Mitmachen denn als Teilhabe - auch an politischer Macht - zu verstehen.

Das ist keine platte Verzweckung gesellschaftlicher Kräfte für staatliche Ziele. Sehr 
wohl wird gesehen, dass die Zivilgesellschaft auch der Ort ist für Diskurs und Verständigung, auch für Konflikte und Proteste, dass bürgerschaftliches Engagement der Unabhängigkeit bedarf sowohl vom Staat als auch vom Markt. (8) Aber die Orientierung an den Potenzialen rückt deutlich die »positiven « Wirkungen und Funktionen bürgerschaftlichen Engagements und der Zivilgesellschaft in den Vordergrund. Die integrierenden und kompensierenden Leistungen des zivilgesellschaftlichen Engagements, die Notwendigkeit zur Hebung der betreffenden Potenziale sind derart offensichtlich, dass bürgerschaftliches Engagement fast schon zur Staatsbürgerpflicht wird. Oder wie soll man verstehen, dass der Koalitionsvertrag unter der Überschrift »Ehrenamt « die Erwartung äußert, dass El-
Hier lohnt die Rückbesinnung, dass die Zivilgesellschaft Freiheitsrechte zum Fundament hat, die dem Staat abgetrotzt werden mussten und als Abwehrrechte gegen den Staat auch im demokratischen Gemeinwesen nach wie vor ihren Sinn haben. Die Zivilgesellschaft ist selbstverständlich nicht nur die Gesellschaft der Wohlanständigen und Konstruktiven. Sie ist auch die Gesellschaft derer, die die gesellschaftlichen Analysen staatlicher Stellen nicht teilen, sich nicht an der Stabilisierung dessen beteiligen wollen, was sie nicht für zukunftsfähig halten und wogegen sie aktiv Widerstand leisten. Sie ist auch die Gesellschaft derer, die sich vom Staat, auch von dominierenden zivilgesellschaftlichen Organisationen emanzipieren wollen - nicht im Namen der Potenziale, sondern der Freiheit.

\section{"Zur Zivilgesellschaft gehören auch die Menschen, die sich vom Staat und von großen Organisationen abwenden - im Namen der Freiheit"}

tern ihre Rechte und Pflichten wahrnehmen? (9) War die Familie bisher nicht eine Lebensform, die eher auf die Unterstützung der Zivilgesellschaft rechnen konnte? Soll sie nun in die Reihe der Potenzialträger eingeordnet und an ihre (ehrenamtlichen) Pflichten erinnert werden? Oder wie ist das Bild von Ministerin von der Leyen zu verstehen, wenn sie in einer Talkshow vom langen Arm des Staates spricht, der in die feingliedrigen Finger der zivilgesellschaftlichen Organisationen ausläuft?

Die aktuellen, nicht zu leugnenden gesellschaftlichen und staatlichen Herausforderungen und die durchaus berechtigte Erwartung an die Zivilgesellschaft und ihren eigenständigen Beitrag lassen sie offenbar vorrangig als $\mathrm{zu}$ öffnenden »Ressourcenspeicher « erscheinen. Neben Integration, Kompensation und Partizipation kann eine wesentliche vierte Dimension zivilgesellschaftlichen Handelns aus dem Blick geraten: die Emanzipation. Die Erfolgsberichte über die bisherige Engagementpolitik erwecken bisweilen den Eindruck, diese Politik wende sich vor allem - wenn nicht ausschließlich - an die gutwilligen und konstruktiven Bürgerinnen und Bürger, die die Sicht der staatlichen Stellen auf die Herausforderungen weitgehend teilen und sie mit durchaus eigenen und innovativen - Lösungen bewältigen helfen wollen.
Hier wird eine Spannung deutlich, die nicht mit einer allzu konstruktiven Sicht der Zivilgesellschaft überspielt werden darf. Zu Recht weisen Haas und Treber darauf hin, dass eine Bürgergesellschaft mehr ist als die unterschiedlichen, je in sich legitimen Formen des Bürgerengagements, mehr als Philanthropie oder die freiwillige Mitarbeit in Handlungsfeldern von Politik und Wohlfahrt, die der Staat nicht ausgestalten will oder kann. (10) Bürgergesellschaft ist der Raum der öffentlichen Auseinandersetzung um eine gerechte und gute Gestaltung der Gesellschaft und keineswegs nur ein Projekt der bürgerlichen Elite. So nützlich, sinnvoll und legitim der Beitrag bürgerschaftlichen Engagements zu einer besseren Lebensqualität ist, geht es noch um mehr, nämlich um die Zukunft der Demokratie als Lebensund nicht nur als Regierungsform.

Große Bedeutung kommt unter diesem emanzipatorischen Aspekt deshalb gerade solchen legitimen, wenn vielleicht auch störenden Formen zivilgesellschaftlichen Engagements zu, die nicht nur auf Mitmachen, sondern auf gestalterische Teilhabe auch an politischer Macht setzen. Dazu gehören beispielsweise Formen des Community Organizing, die um die Freiheit der Auseinandersetzungen mit der Kommunalpolitik willen auf staatliche Förderung bewusst verzichten, weil sie die schleichende
Monetarisierung ihres Engagements vermeiden und der Abhängigkeit entgehen wollen.

Ein solcher emanzipatorischer Ansatz scheint quer zu liegen zu einer Engagementpolitik als einer Förderpolitik für bürgerschaftliches Engagement. Wie soll gefördert werden, was nicht der Förderrichtung entspricht oder sich der Förderung gar verweigert? Wie soll Gegenstand der politischen Steuerung und Gestaltung sein, was gerade die Unabhängigkeit und Freiheit von solcher Gestaltung sucht? Kann nur Gegenstand der Engagementpolitik sein, was im parlamentarischen Verfahren durch Fördergesetze als förderungswürdig festgeschrieben wurde - oder kann paradoxerweise staatliche Engagementpolitik die Emanzipation vom Staat gutheißen und fördern?

\section{"Anerkennungskultur" reicht nicht}

Hier lohnt ein Blick auf die immer wieder geforderte »Anerkennungskultur «. Bei der Anerkennung des Ehrenamtes und anderer Formen des zivilgesellschaftlichen Engagements mögen Ehrenamtsausweise, Anerkennungsschreiben und Feierstunden unverzichtbar sein. Noch mehr als Anerkennung braucht dieses Engagement aber Respekt. Respekt ist eine Form der Anerkennung, die gerade nicht nur das aktuell politisch »Konstruktive « wertschätzt, sondern auch im Widerständigen, Störenden die Verwirklichung von Freiheits- und Bürgerrechten erkennt, die demokratische Kultur fördert - gerade auch wenn dies in den Konflikt führt.

Noch wichtiger als ein Förderprogramm ist für die Gewährung solchen Respekts beispielsweise eine durchgängige Politik und ein entsprechendes Verwaltungshandeln, die sich aktiv ansprechbar, auffindbar und antreffbar zeigen für die zivilgesellschaftlichen Initiativen, die die Spannung zwischen gesellschaftlichen und staatlichen Anliegen nicht als Störung, sondern als produktive Kraft und demokratische Notwendigkeit begreifen. Politiker sehen dann beispielsweise in Engagierten nicht bloß rührig Tätige, denen man lediglich bei Festveranstaltungen begegnet, oder gar Störer, die liebgewordene Muster hinterfragen und deren Kontakt man besser meidet, sondern Bürgerinnen und Bürger, deren kritischer Beitrag für die Politik im Gemeinwesen konstitutiv ist und denen ein gleichrangiger Platz auf der politischen Agenda und im Terminkalender zukommt wie gewichtigen Kräften aus Staat und Wirtschaft.

Respektvolle Anerkennung zivilgesellschaftlichen Engagements wird nicht immer in bereits strukturierten und eingeführten Verfahren erfolgen können. Nimmt 
Verwaltung die kreative und innovative Funktion dieses Einsatzes ernst, wird sie zusätzliche, nicht ungesetzliche, aber außergesetzliche Beteiligungsformen erwägen und vorsehen. Es reicht nicht, eine Checkliste mit verpflichtenden Beteiligungen durchzugehen. Respekt fordert vielmehr ein wachsames Interesse und aktives Zugehen auf Gruppen und Initiativen.

Solche "Förderung und Anerkennung " des zivilgesellschaftlichen Engagements ist möglich außerhalb von formellen Förderprogrammen, leistet keiner Monetarisierung Vorschub und ist mit der emanzipatorischen Dimension des Engagements vereinbar, ja fördert sie. Denn so kann auch das »Kritik- und Störpotenzial « abgeholt werden, das in einer dialektischen Weise staatliches Handeln relativiert und fördert. Denn in der Engagementpolitik geht es nicht nur um die Förderung des Engagements durch den Staat, es geht auch um die Förderung des demokratischen Staatswesens durch eine wache und aktive Zivilgesellschaft - mag sich manches auch heute als Störung darstellen, was morgen als Fortschritt integriert wird.

\section{Anmerkungen}

(1) So sieht es jedenfalls das Wissenschaftszentrum Berlin für Sozialforschung in seinem »Bericht zur Lage und zu den Perspektiven des bürgerschaftlichen Engagements in Deutschland ", Hg.: Bundesministerium für Familie, Senioren, Frauen und Jugend, Berlin 2009, S. 12.

(2) Bundesministerium für Familie, Senioren, Frauen und Jugend ( $\mathrm{Hg})$ : Engagementpolitik wirksam gestalten, neue Impulse für die Bürgergesellschaft ein Jahr Initiative ZivilEngagement, Berlin 2008, S. 12.

(3) Engagementpolitik wirksam gestalten, S. 2.

(4) Wissenschaftszentrum Berlin, S. 5.

(5) Wissenschaftszentrum Berlin, S. 18.

(6) Engagementpolitik wirksam gestalten, S. 16.

(7) Engagementpolitik wirksam gestalten, S. 27.

(8) Wissenschaftszentrum Berlin, S. $11 \mathrm{f}$.

(9) Koalitionsvertrag, S. 80.

(10) Haas, Hans Stephan, Treber, Monika (Hg.): Enabling Community - Gemeinwesen zur Inklusion befähigen, Hamburg/Berlin 2009, S. 11.

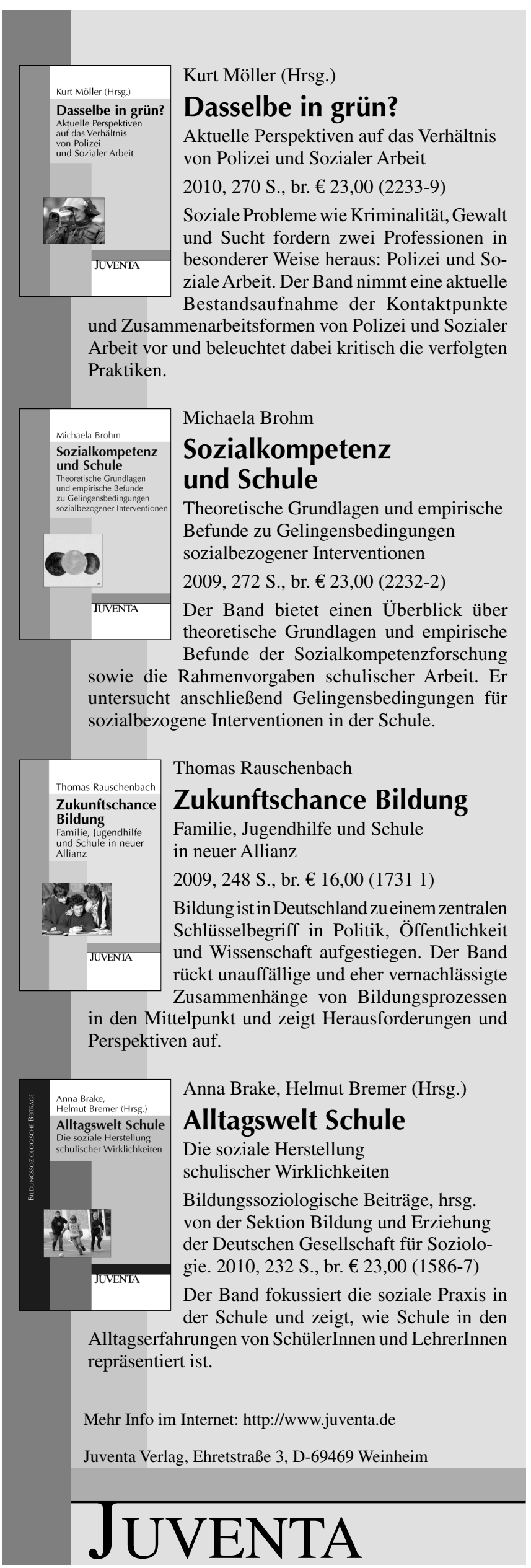

